\title{
A Study on Promotion Strategies of Clinical Teachers' Teaching Ability in Affiliated Hospitals
}

\author{
Chunmiao Liu \\ Higher Education Research and Teaching Quality Evaluation Center, Faculty Development Center, Qiqihar \\ Medical University, Qiqihar City, Heilongjiang Province, 161006, China \\ Email:29020230@qq.com
}

Keywords: affiliated hospital; clinical teaching; faculty; teaching ability; promotion strategy

\begin{abstract}
Affiliated hospitals of medical colleges and universities are the main practice places for medical colleges and universities, which play a tremendous role in promoting the progress of medical education. With the improvement of the social, economic and cultural level, people have put forward higher requirements for the quality of treatment and service of clinical medicine. However, due to the influence of the reform of China's medical and health system and various factors of medical personnel, the clinical teachers' teaching ability in affiliated hospitals still faces some problems. Based on the author's learning and clinical experience, this article first analyzes the current situation of the clinical teaching staff in affiliated hospitals, and then proposes strategies for improving the teaching ability to enhance the quality of clinical practice teaching.
\end{abstract}

\section{Introduction}

With the expansion of the medical education scale, the medical resources appear to be inadequate. Due to the reform of the medical system, the implementation of the Law for Licensing Medical Practitioner and the enhancement of the patient's self-protection awareness, the training of medical students' clinical practical ability has gradually become a weak link in medical education. How to take effective measures to improve teachers' teaching ability in affiliated hospitals and enable students to receive good clinical practice skill training within a limited time is an important issue confronted by medical colleges and is a new task faced by affiliated hospitals.

\section{Current Situation of the Clinical Teaching Staff in Affiliated Hospitals}

\subsection{Poor management system and weak teaching awareness}

Clinical teachers in affiliated hospitals shoulder the dual tasks of medical care and teaching. They are both front-line clinical medical workers and teaching workers. Because of requirements for professional title promotion, they must also conduct scientific research with busy work. Since there is no administrative affiliation relationship between indirectly affiliated hospitals and medical colleges, the personnel and financial affairs between the two are independent with no interference to each other. It is difficult for medical colleges to formulate perfect restrictions and incentives for clinical teaching in indirectly affiliated hospitals. Therefore, clinical teachers do not actively engage in clinical teaching. Moreover, if there are serious teaching accidents among clinical teachers who are responsible for teaching tasks, it is difficult for medical schools to handle them in accordance with relevant regulations. In the long run, it may easily lead to a weak teaching awareness among clinical teachers and they will lack initiative and responsibility for teaching work. When there is a conflict between medical treatment and teaching, teaching will often give way to medical treatment. In this way, it is easy to form a kind of atmosphere where the teaching work is the same as non-teaching work and good teaching work is the same as the bad teaching work within the hospital. This will weaken the position of clinical teaching in the overall work of the hospital, which will lead to a serious decline in the quality of clinical teaching and make it difficult to improve students' clinical practice ability. 


\subsection{Lack of basic teaching skills and difficulty in improving teaching}

Clinical teachers have solid medical theory knowledge and rich clinical practice experience. Classroom teaching can explain theories with a large number of clinical practices. The diverse teaching forms can explain the profound in simple terms and this will achieve good teaching results. However, pre-vocational education for clinical teachers is subject to the education of medical disciplines and humanistic qualities. Besides, their inductive education is subject to short-term training in pedagogy, psychology, and educational laws and regulations. When they enter the workplace, clinical teachers spend most of the time on clinical medicine work and they lack a comprehensive and systematic grasp of pedagogic theoretical knowledge and basic teaching concepts. Therefore, it is difficult for them to take special time for systematic study or training in busy medical and teaching work, and to apply advanced educational concepts to clinical teaching practice. Their ability to use the language skills of teaching, master teaching difficulties, choose teaching materials and content, and handle teaching emergencies still needs to be improved. These factors affect the teachers' teaching level, so an excellent clinician may not be an excellent clinical teacher. Only when clinicians possess a solid theoretical knowledge of education and teaching, and receive systematic and rigorous training, and are diligent in learning and good at summary, can they quickly become an excellent clinical teacher.

\subsection{Ineffective teacher training measures and difficulty in improving teaching ability}

Due to the growing requirements of discipline and specialty for medical practice in medical education, the cultivation of clinical teachers should establish a long-term mechanism and focus on sustainable development goals. From a realistic point of view, medical colleges and indirectly affiliated hospitals have a cooperative teaching relationship and affiliated hospitals also undertake certain teaching tasks. In fact, the affiliated hospitals do not form an atmosphere that attaches importance to teaching. Clinical teaching is an "additional task" for affiliated hospitals or just a task to meet the need for promotion of teaching titles. Teacher training is less valued than medical services. It lacks effective management and promotion mechanism which is a short-term behavior. When the clinical teacher undertakes the task of teaching, they may temporarily accept the guidance of teachers with long past service in the same department or spend some time to participate in teaching observation and peer lectures. A small number of teachers directly participate in the classroom teaching with their own understanding and experience. After the teaching tasks are completed, they will continue to engage in medical work and leave the teaching work behind. The same is true for the next round of teaching arrangements. Teachers' teaching ability lacks continuous and effective training, and it is difficult to improve their teaching ability.

\section{Promotion Strategies of Teaching Ability}

\subsection{To improve clinical teaching admittance standards for teachers}

The first is to strengthen the construction of teachers' morality and work style, and promote innovation in cultural heritage. The school should strengthen professional ideals and ethics education, vigorously promote the advanced deeds of college teachers and moral models, and guide clinical teachers to devote themselves to teaching and educating people. In teacher training, especially pre-vocational training for new teachers and the ethics of clinical doctors should be strengthened. The uniqueness of the medical profession requires that clinical teachers have a higher self-cultivation and need to continuously strengthen the study of humanistic knowledge. The second is to improve the academic standards of teachers. The responsibility of clinical teachers lies in teaching clinical students the correct clinical knowledge and skills, cultivating their interest in medical research, and laying the foundation for the cultivation of high-quality medical professionals in the future. 


\subsection{To apply diversified training methods to improve the comprehensive capabilities of clinical teachers}

The first is to strengthen basic professional skills training. In order to improve the professional level and teaching ability of clinical teachers, the hospital needs to improve the basic teaching organizations, such as teaching and research rooms, teaching teams and curriculum groups, to persist in collective lesson preparation and to deepened the research on key and difficult teaching issues. It can use lectures, teaching observation and other forms to invite excellent teachers in domestic and overseas hospitals to teach teaching skills and practice lessons. Besides, teachers who received the first prize in the teaching competitions can conduct demonstration on-site lesson. The second is to strengthen teaching ability training. The hospital needs to cultivate the teachers' ability to independently engage in teaching and scientific research work, and to provide them with an exchange platform both inside and outside the school, so that they can learn excellent teaching experience, scientific thinking and innovative ability from the old and new colleagues. Teachers are encouraged to participate in various large-scale teaching conferences, to exchange conference papers and to speak at the conference. After the conference, discussions are held with other institutions to broaden their horizons and increase their knowledge.

\subsection{To adopt various incentive measures to promote the improvement of teachers' ability}

The first is to encourage teachers to participate in teaching competitions and micro-teaching competitions. Each contest includes special awards, first prizes and second prizes. Special awards will be allowed for vacancies and outstanding individuals can participate in provincial and national competitions. This method has a great impact on teaching, which not only mobilizes the enthusiasm of teachers, but also improves the teacher's teaching skills to make teaching more exciting. The second is to encourage teachers to participate in teaching courseware production. The competition aims to promote teachers' excellence in courseware production to attract students. At the same time, teachers are required to pay attention to the collection of teaching materials, such as photographs and typical cases in their work. These teaching materials should be used in courseware production, so that clinical work can be closely integrated with clinical teaching. The third is to increase the proportion of teaching tasks in the promotion of professional titles. All kinds of teaching honors, awards and papers for teachers can be appropriately scored during promotion, which effectively promotes the improvement of the teaching level of teachers.

\subsection{To strengthen the mastery and application of new teaching methods and technology}

The first is to master a variety of teaching methods. The hospital may use a variety of teaching methods, integrate related courses, and strengthen the training of learning methods for medical students to improve their core competitiveness, which will help students adapt to clinical teaching as soon as possible. Problem-based teaching methods and problem-oriented discussions around clinical cases have greatly improved the medical students' initiative in learning, cultivated students' logical reasoning ability and promoted the medical humanities and literacy. Standardized patient teaching can train medical students to collect medical history comprehensively and systematically, and strengthen medical students' ability to communicate with doctors and patients. Besides, using simulation technology to teach and repeat operations on simulated persons and organs reduces the psychological burden on medical students, reduces medical risks and cultivates students' learning interest and basic ability to make a correct diagnosis and treatment of the disease. Moreover, as the mainstream methodology representing the current medical practice, evidence-based medicine (EBM) has gradually become the latest thinking model for the treatment of diseases and medical decisions. The practice of EBM can tell medical students that in addition to clinical experience, they should focus on the use of the best obtained medical documentary evidence, take into account of the patient's value and desire, and make reasonable clinical decisions for the patient.

The second is to use new technology to improve teaching efficiency. Teachers can publish their course videos, courseware and reference materials on the Internet. Students can choose to study independently. The large-scale open network course is a new type of teaching method that combines 
information technology, network technology and quality education. Due to the openness, networking and initiative of MOOC teaching, it greatly mobilizes the enthusiasm of students.

The third is to use new technology to improve the effectiveness of teaching evaluation. Objective structured clinical examination (OSCE) is an internationally popular form of clinical competence assessment for clinical medical students and junior doctors, which uses multiple-step assessment methods to conduct fair and objective evaluation of the candidate's clinical skills with standardized patients, virtual patients, computers and other means. The application of the OSCE model helps to objectively evaluate clinical teachers' clinical skills and effect.

\section{Summary}

The vast majority of teachers in medical colleges and universities are clinical doctors in affiliated hospitals, and their teaching ability directly affects the overall teaching quality of medical schools. Due to the way of work, some teachers' clinical teaching skills need to be further improved. Therefore, it is necessary to adopt multiple angles and all-round measures to improve the teaching capacity of clinical teachers, including improvement of teachers' clinical teaching admission standards, application of multiple training methods to improve comprehensive ability, use of appropriate incentive measures and application of effective evaluation system. This will urge teachers to improve their teaching abilities and help teachers develop appropriate teaching plans simultaneously to apply new technology and new teaching methods to clinical teaching.

\section{Acknowledgement}

2017 record subject of the 13th Five-Year Plan of Educational Science in Heilongjiang Province(GBD1317150).

\section{References}

[1] Ma Xincui, Li Baohua, Tian Yi. A Study on Experience and Thinking of Clinical Nursing Teaching Supervision and Assessment Process [J]. China Medical Education Technology, 2018, (01): 98-102.

[2] Zhang Yanhu, Zhu Pinghui, Guo Xu, Ma Dongmei. An Investigation of Clinical Teaching Ability of a Three-level General Hospital in Beijing [J]. Continuing Medical Education, 2017, (05): 40-42.

[3] Xu Yun, Xie Xiaohua, Tan Wei, Hong Pu, Chen Yuanyuan, Zhao Jiangli, Zhao Lihong, Li Dongru, Wang Jiaying. A Study on Evaluation and Countermeasures of Clinical Nursing Teachers' Teaching Ability at the Undergraduate Level [J]. Chinese Nursing Research, 2017, (06): 729-731.

[4] Yang Shilai, Wang Xiaoxia. A Research on the Progress of Clinical Nursing Teaching [J]. Journal of Nursing, 2015, (24): 34-36.

[5] Liu Zhijing, Wang Xiuchun. A Study on the Cultivation of Teaching Ability for Teachers in Clinical Medicine [J]. World Latest Medicine Information, 2015, (10): 183.

[6] Zhang Fuzhi, Liu Ying, Lu Zhaojun. A Discussion on the Training Path of Clinical Teachers [J]. Journal of Liaoning Medical University (Social Science Edition), 2014, (04): 27-29.

[7] Pan Yingli, Tan Jianling. A Research on the Teaching Ability of Clinical Teachers [J]. Chinese Nursing Research, 2012, (11): 981-982. 